\title{
Controle ativo De Ruído E Vibrações Mecânicas Utilizando um algoritmo de Controle tipo DEADBEAT
}

\author{
JoSÉ MARIA GALVEZ \\ Departamento de Engenharia Mecânica, Universidade Federal de Minas Gerais, Brasil. Av. Antônio Carlos 6627, \\ Pampulha, 31.270-901 Belo Horizonte, MG, Brasil. \\ jmgalvez@ufmg.br
}

\begin{abstract}
In the area of active control of noise and mechanical vibrations, the appropriate strategy to be used is always dependent of the control objective. The solution of the control problem is given for each case, any situation will require a specific engineering solution, turning this area a vast field of theoretical and practical research. As a consequence of the steadily improvement of digital computers speed performance in the last decades, several new digital control strategies have been proposed by the scientific community, among them, the deadbeat control strategy, successfully proposed for regulation type problems. This article presents an extension of the technique of deadbeat control in the noise reduction and control of mechanical vibrations case. Initially, the design procedure of the proposed control strategy is presented and discussed. It is shown that for a sampling rate sufficiently fast, the deadbeat controller delivers a fast and precise response. Finally, simulation results to verify the viability of the proposed technique and to evaluate the controller performance are presented.
\end{abstract}

Keywords - Noise control, Vibration Control, Deadbeat Control.

Resumo- Na área de controle ativo de ruído e vibrações mecânicas, a estratégia utilizada é sempre dependente do objetivo de controle. A solução do problema de controle é dada caso a caso, cada situação irá requerer uma solução particular de engenharia tornando esta área um vasto campo de pesquisa teórica e prática. Como conseqüência do continuo aprimoramento da velocidade de processamento dos computadores digitais acontecido nas últimas décadas, várias novas estratégias de controle digital tem sido propostas pela comunidade científica, entre elas, a estratégia de controle deadbeat. Inicialmente proposta para solucionar problemas de regulação, neste artigo, a técnica de controle deadbeat e apresentada como uma solução alternativa do problema de redução de ruído e controle de vibrações mecânicas. Inicialmente, o procedimento de projeto do algoritmo proposto é apresentado e discutido. É mostrado a seguir que para uma taxa de amostragem suficientemente rápida, o controlador deadbeat fornece uma resposta de controle rápida e precisa. Finalmente, resultados de simulação são apresentados para verificar a viabilidade da técnica proposta e avaliar o desempenho de controlador.

Palavras chave — Controle de Ruído, Controle de Vibrações, Controle Deadbeat.

\section{Introdução}

A qualidade de vida se tornou a preocupação principal entre a população das sociedades modernas. Em face à recente enorme expansão de áreas industriais, manter saudáveis os ambientes domésticos e de trabalho se tornou um objetivo importante dos engenheiros de planejamento urbano $\mathrm{e}$ industrial. Um elemento invisível, mas não menos importante, de poluição de ambientes é o ruído e/ou vibração mecânica produzidos por uma variedade enorme de fontes. Ruído e vibração em ambientes são responsáveis por doenças físicas e psicológicas entre humanos, trazendo perdas econômicas significativas às linhas de produção, Barron (2003). Para lidar com ruído e vibrações indesejados, duas linhas de pesquisa podem ser identificadas; elas são conhecidas como: técnicas de controle ativo que tiveram seu maior desenvolvimento junto com a moderna automação industrial e técnicas passivas de controle existentes desde trabalhos pioneiros como o de Cremer (1953) e outros.

Esquemas de controle ativo são apropriados para situações com ruído de baixa freqüência. Beranek (1992). Nesta faixa de freqüência eles são mais eficientes e de menor custo do que esquemas passivos. Em alta freqüência, os sistemas passivos de controle de ruído são ainda a escolha mais apropriada. Técnicas de controle passivas incluem isoladores, silenciadores, sistemas de amortecimento de vibração, dispositivos de absorção, etc. Técnicas passivas trabalham melhor em altas freqüências e elas desenvolvem um papel importante em quase qualquer maquinaria industrial, comercial ou doméstica no mundo atual. Porém, quando usado em aplicações de baixas freqüência, as técnicas passivas envolvem normalmente equipamento vultoso e pesado. Isto porque o tamanho e massa dos dispositivos passivos dependem do comprimento de onda acústico, tornando-se mais pesados e volumosos em baixas freqüências. $\mathrm{O}$ controle de ruído e vibrações de baixa freqüência tem sido tradicionalmente difícil, caro e em muitos casos inviável através de técnicas passivas por causa dos longos comprimentos de onda envolvidos. Por outro lado, o tamanho pequeno e o peso leve dos equipamentos envolvidos das técnicas de controle ativo se tornaram um fator extremamente importante, e em muitos casos, um benefício insubstituível.

O controle ativo de ruído/vibração consiste no cancelamento do campo acústico de ruído/vibração através de meios de eletromecânicos. Neste caso, o sistema de controle aciona um atuador (caixa acústica ou fonte de vibração) para produzir um campo acústico que é uma imagem em oposição de fase do campo indesejado (do ponto de vista de controle isto pode ser visto como um cancelamento pólo-zero da planta). $\mathrm{O}$ resultado é a redução do ruído e/ou vibração. A idéia de usar controle ativo de ruído como uma alternativa para o controle passivo em baixas freqüências foi inicialmente proposto por Paul Lueg em 1933. A idéia 
básica era usar um transdutor para introduzir uma perturbação secundária no sistema e assim cancelar o ruído primário existente. Durante várias décadas, a ausência de tecnologia adequada e conhecimento insuficiente na área de controle manteve estas idéias em suspense. Somente na década de 80, o controle ativo de ruído se tornou novamente o foco de interesse da comunidade científica. Naquele tempo o surgimento da tecnologia de pequenos computadores e outros avanços em engenharia de controle tornaram possível o implementação dos primeiros sistemas de controle ativo de ruído. A nova tecnologia na área de sensores e atuadores desenvolvida nas últimas décadas do século $\mathrm{XX}$, assim como, o custo decrescente de computadores de pequeno porte já existentes, levaram a um imenso crescimento da pesquisa neste campo com algumas aplicações comerciais interessantes implementadas recentemente com sucesso.

Este artigo é organizado como segue: Na Seção 2, o problema de controle ativo de ruído é comentado. $\mathrm{Na}$ Seção 3, uma breve revisão da técnica de controle digital deadbeat é apresentada. $\mathrm{Na}$ Seção 4, o procedimento de projeto do controlador deadbeat aplicado à redução de ruído e vibrações é considerado. $\mathrm{Na}$ Seção 5, resultados de simulação para ilustrar o desempenho do esquema de controle proposto são apresentados. Finalmente, na Seção 6, são apresentadas comentários finais e conclusões do trabalho.

\section{O Problema de Controle Ativo de Ruído}

Os modernos sistemas de controle ativo de ruído consistem em uma ou mais fontes acústicas controladas utilizadas para introduzir um sinal secundário no sistema acústico. O sinal introduzido suprime o ruído indesejado originado pelas fontes primárias de ruído. $\mathrm{O}$ algoritmo de controle, normalmente implementado em um controlador digital, utiliza como entradas as medições do ruído produzidas pelas fontes primárias a partir dessa informação o algoritmo gera o sinal de controle para as fontes acústicas controladas, Hansen (1996, 1997).

Um item importante para garantir o bom desempenho do sistema de cancelamento de ruído é o projeto satisfatório da estrutura acústica do sistema, Beranek (1992). Tendo alcançado uma boa solução para a estrutura acústica, um segundo passo é a escolha adequada do hardware para o controlador digital que implementará o algoritmo de controle. Além disso tudo, a especificação e posicionamento dos atuadores, sensores e transdutores é fundamental para atingir os objetivos de projeto.

Técnicas de controle ativas de ruído são a escolha apropriada para campos de ruído que são espacialmente simples tais como ruído de baixa freqüência se propagando ao longo de uma estrutura tubular. Os controladores ativos de ruído alcançam seus melhores desempenhos quando o comprimento de onda for mais longo que as dimensões de seu ambiente de propagação o que caracteriza um problema unidimensional. Um pior caso seria uma geometria complexa envolvendo ruído de alta freqüência de banda larga, isto é , ruído que contenha uma gama extensa de freqüências. Este caso é mais difícil de controlar que ruído de banda estreita que contenha uma forte freqüência fundamental.

Diversas aplicações de controle ativo de ruído podem ser encontradas na indústria: controle ativo de vibração e ruído em aeronaves como helicópteros, navios e submarinos; redução de ruído de baixa freqüência em ambientes industriais como bombas de vácuo, sopradores de ar, torres de refrigeração e exaustores de gás; controle ativo de vibração de baixa freqüência em estruturas espaciais; controle ativo de oscilações em edifícios altos; redução ativa de ruído em sistemas de ar-condicionado e em transformadores elétricos de grande porte; redução de ruído em cabinas de automóveis; sistemas de suspensão dinâmicos para veículos; auriculares ativos para pilotos de aeronaves; e outras, Barron (2003), Crolla et al (1991), Cuesta et al (2000), Donadon et al (2005), Fujita (1997), Hansen(1996), Silvester (1966), Stein et al (1991).

A estratégia apropriada de controle utilizada é sempre dependente do objetivo de controle, isto é, se é controle de vibração, controle de potência de som irradiado, controle de transmissão de som ou algum outro objetivo. Por exemplo, a estrutura física de controle por reduzir ruído no interior de aeronaves não é igual à estrutura física necessária para reduzir a transmissão de ruído num tubo de condução de ar ou num sistema para isolamento de vibrações em um microscópio eletrônico. Da mesma forma, o controlador eletrônico para um sistema de controle ativo tipo feedforward (Cabell et al, 1999) não será igual a aquele controlador eletrônico para um sistema tipo realimentação de saída. Porém, os princípios básicos de projeto eficiente para cada aplicação serão os mesmos, Hansen (1996).

$\mathrm{Na}$ área de controle ativo de ruído e de vibrações mecânicas, a estratégia utilizada é sempre dependente da estrutura acústica e do objetivo de controle. A solução do problema de controle é obtida caso a caso, cada situação irá requerer uma solução particular de engenharia tornando esta área um vasto campo de pesquisa teórica e prática.

\section{A Técnica Deadbeat - Breve Revisão}

A flexibilidade, baixo custo, capacidade computacional e velocidade dos novos computadores digitais tem permitido o desenvolvimento de novos algoritmos de controle que não são simplesmente uma versão discreta de controladores analógicos. Em particular, é possível formular novas leis de controle que, em condições adequadas, produzirão a resposta de malha fechada desejada. Os componentes principais de um sistema de controle em malha fechada são: A planta que é o sistema físico a ser controlado; exemplos 
típicos em controle ativo de ruído são auriculares para uso aeronáutico, o ar que percorre o interior de um duto de ar-condicionado. Os sensores que são dispositivos, como microfones e acelerômetros, usados para medir a perturbação. Os atuadores que são dispositivos que fisicamente executam a tarefa de modificar a resposta de planta; eles normalmente são dispositivos de eletromecânicos como caixas de som ou geradores de vibração (shakers). E finalmente o algoritmo de controle que define a potência a ser fornecida à planta pelo atuador e que pode ser implementado em computadores digitais, microcontroladores ou qualquer dispositivo digital capaz de executar algoritmos numéricos, Elliott et al (1992), Fuller et al (1995), Kuo et al (1996), Nelson et al (1992). O diagrama de blocos padrão de um sistema de controle digital é mostrado na Figura 1. A Figura 2 mostra o diagrama de bloco simplificado do sistema em malha fechada.

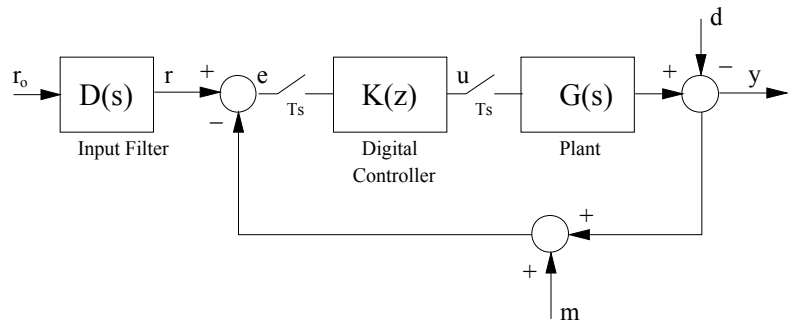

Figura 1. Diagrama de Blocos do Controlador Digital,

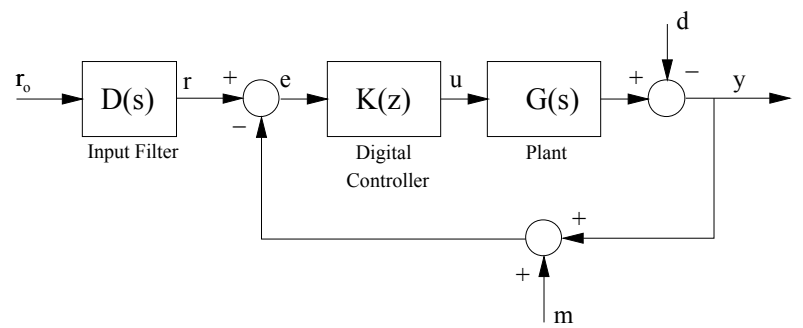

Figura 2. O Diagrama de Blocos Simplificado.

Neste caso d(t) é uma perturbação, m(t) é um ruído de medição, $r(t)$ representa a saída desejada (referencia), e(t) é o sinal de erro usado pelo algoritmo de controle para determinar $\mathrm{u}(\mathrm{t})$ que corresponde à potência a ser fornecida à planta. $\mathrm{D}(\mathrm{s})$ é a função de transferência no domínio de Laplace do filtro utilizado para suavizar mudanças bruscas no sinal de referência, G(s) é a função de transferência no domínio de Laplace da planta e finalmente $K(z)$ é a função de transferência no domínio $\mathrm{Z}$ que representa o algoritmo de controle. A função de transferência de malha fechada, $T(z)$, da entrada $\mathrm{r}$ para saída y no domínio $\mathrm{Z}$ é dada por:

$$
T(z)=\frac{Y(z)}{R(z)}=\frac{G(z) K(z)}{1+G(z) K(z)}
$$

O objetivo do controle deadbeat é atingir a melhor resposta possível a uma mudança na referencia. $\mathrm{Na}$ teoria de controle, o projeto do controlador deadbeat consiste em determinar um sinal de controle que aplicado à planta leva a saída para zero no menor número de intervalos de amostragem, Chen (1994), Dorf et al (2005), Kailath (1980). Pode ser mostrado que para um sistema linear invariante no tempo de ordem $\mathrm{n}$ este número de intervalos de amostragem é $\mathrm{n}$, desde que o sistema seja "null controlable" (possa ser levado para origem por alguma entrada finita). Isto pode ser alcançado determinando um controlador, $\mathrm{K}(\mathrm{z})$, tal que todos os pólos da função de transferência de malha fechado desejada, $\mathrm{T}_{\mathrm{d}}(\mathrm{z})$, estejam alocados na origem do plano $\mathrm{Z}$. O projeto pode ser levado a cabo utilizando a equação de síntese definida por:

$$
K(z)=\frac{1}{G(z)}\left[\frac{T_{d}(z)}{1-T_{d}(z)}\right] ; T_{d}(z)=z^{-p}
$$

Note que, a função de transferência em malha fechada $T_{d}(s)$ correspondente possui $p$ pólos no infinito. A seguir alguns fatos importantes dos controladores tipo deadbeat, Chen (1994), Dorf et al (2005), Kailath (1980): a) No procedimento de projeto o usuário especifica a resposta de malha fechada desejada, $\mathrm{T}_{\mathrm{d}}(\mathrm{z})$. A técnica de projeto utiliza o inverso do modelo de planta, G(z) que é utilizado para cancelar a dinâmica não desejada da planta (substituindo-a por outra característica dinâmica) de forma que a resposta de malha fechada desejada possa ser alcançada. b) Entretanto, o uso do modelo inverso apresenta um problema quando a planta possui zeros fora do círculo unitário (sistema de fase não mínima); se $G(z)$ tem zero(s) fora do circulo unitário, o controlador $\mathrm{K}(\mathrm{z})$ terá polo(s) fora do círculo unitário e o produto $\mathrm{G}(\mathrm{z}) \mathrm{K}(\mathrm{z})$ terá um cancelamento de polo/zero na região instável, neste caso, a equação de síntese não deve ser utilizada. c) Se a resposta de malha fechada desejada, $T_{d}(z)$, for escolhida mais rápida do que a do sistema real o controlador pode se tornar não-causal. d) Pode ser mostrado que quando a taxa de amostragem aumenta, sistemas contínuos de fase mínima fase podem se tornar sistemas discretos de fase não-mínima no domínio $\mathrm{Z}$; isto usualmente acontece quando o sistema contínuo tem um excesso de (número de pólos - número de zeros) maior que 2. e) A escolha de uma taxa de amostragem muito rápida - sem modificar a resposta de malha fechada desejada $T_{d}(z)$ - tem como conseqüência um controlador com desempenho brusco entre instantes de amostragem. f) O fenômeno conhecido como "ringing" pode ser causado por pólos do controlador com parte real negativa; quanto mais próximo do ponto -1 no plano $\mathrm{Z}$, mais grave será $\mathrm{o}$ problema, neste caso, o controlador pode produzir um sinal de controle oscilatório com amplitude decrescente.

\section{O Esquema De Controle Proposto}

A técnica de controle deadbeat tem sido tradicionalmente aplicada para solucionar casos de 
regulação, Chen (1994), Dorf et al (2005), Kailath (1980). Este artigo considera uma extensão das aplicações da técnica deadbeat, neste trabalho, um controlador deadbeat é usado para reduzir perturbação de saída não desejada (note que esta não é a aplicação natural para este tipo de controlador). Pode ser mostrado que para uma taxa de amostragem corretamente escolhida, o controlador deadbeat pode ser utilizado com sucesso ao problema de controle ativo de ruído e vibrações mecânicas de baixa freqüência.

Esta seção apresenta um esquema de controle simples e direto para o problema de redução de ruído e vibrações. O sinal indesejado (ruído ou vibração) é tratado como uma perturbação a um sistema sabido ser estável. Tempos de atraso que eventualmente possam existir neste tipo de sistema, são incluídos naturalmente na formulação da lei de controle; assim como também, os problemas causados pelo posicionamento deslocado de sensores e atuadores. A Figura 3 mostra o diagrama de bloco do esquema de controle proposto. O controlador proposto pode ser visto como um atuador inteligente que fornece o sinal apropriado para cancelar o ruído ou vibração não desejado.

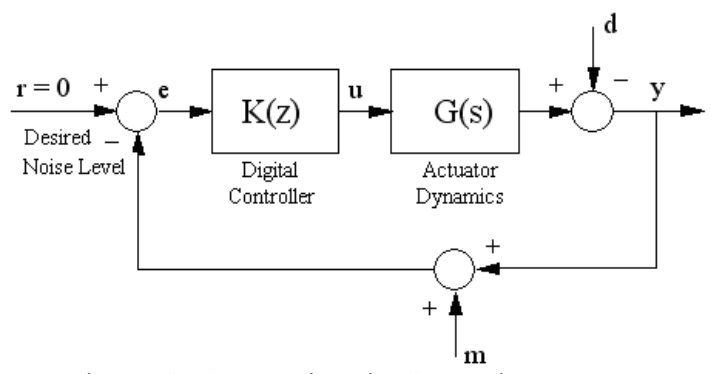

Figura 3. A Técnica de Controle Proposta.

\section{Resultados De Simulação}

Neste trabalho, o modelo de $12^{\mathrm{a}}$ ordem de um guia de onda acústica unidimensional foi utilizado para avaliar o desempenho de controlador. A Figura 4 mostra a resposta ao impulso da planta e a Figura 5 a resposta em freqüência da planta. O modelo utilizado é continuo, linear e de fase mínima cujos pólos e zeros são mostrados na Tabela 1. A freqüência mais baixa da planta é de aproximadamente $50 \mathrm{rd} / \mathrm{s}$ e a mais alta é de $1060 \mathrm{rd} / \mathrm{s}$. Assim, a taxa de amostragem foi escolhida como Ts $=1.5 \mathrm{~ms}$.

Tabela 1. Os pólos e zeros da planta.

\begin{tabular}{|c|c|c|c|}
\hline Pólos & Zeros & Amorte. & Freq. \\
\hline$-5.0 \times 10^{+0} \pm j 5.0 \times 10^{+1}$ & $-1.8 \times 10^{+1} \pm j 1.1 \times 10^{+3}$ & $1.0 \times 10^{-1}$ & $5.0 \times 10^{+1}$ \\
\hline$-1.8 \times 10^{+1} \pm j 2.1 \times 10^{+2}$ & $-2.2 \times 10^{+1} \pm j 6.5 \times 10^{+2}$ & $8.4 \times 10^{-2}$ & $2.1 \times 10^{+2}$ \\
\hline$-1.2 \times 10^{+1} \pm j 6.2 \times 10^{+2}$ & & $2.0 \times 10^{-2}$ & $6.2 \times 10^{+2}$ \\
\hline$-5.7 \times 10^{+1} \pm j 6.7 \times 10^{+2}$ & & $8.5 \times 10^{-2}$ & $6.7 \times 10^{+2}$ \\
\hline$-7.6 \times 10^{+1} \pm j 8.7 \times 10^{+2}$ & & $8.7 \times 10^{-2}$ & $8.7 \times 10^{+2}$ \\
\hline$-2.7 \times 10^{+1} \pm j 1.1 \times 10^{+3}$ & & $2.6 \times 10^{-2}$ & $1.1 \times 10^{+3}$ \\
\hline
\end{tabular}

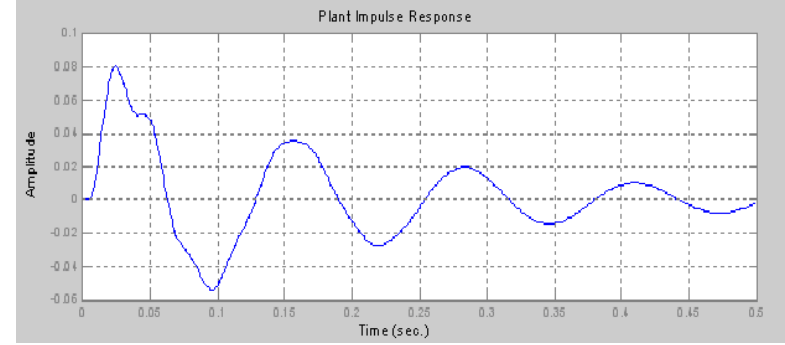

Figura 4. Resposta ao Impulso da Planta.

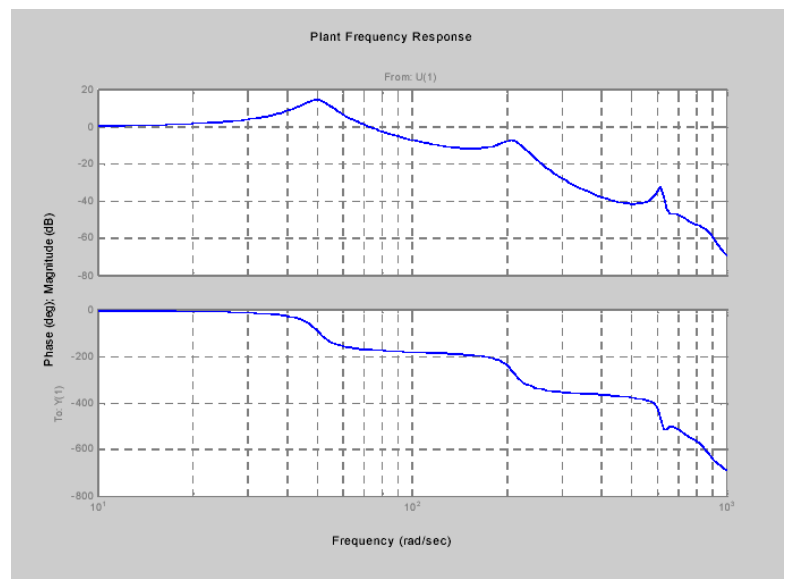

Figura 5. Resposta em Freqüência da Planta.

Para a taxa de amostragem de $1.5 \mathrm{~ms}$, o modelo discreto da planta, $G(z)$, foi determinado incluindo um retentor de ordem zero no caminho direto da malha de controle. Neste caso, G(z) tem um excesso de (número de pólos - número de zeros) de sete. Assim, o controlador foi projetado utilizando a Equação (2) (de forma a obter um algoritmo de controle causal como comentado em Seção 3). A Figura 6 mostra os pólos e zeros da planta e do controlador no domínio $\mathrm{Z}$.

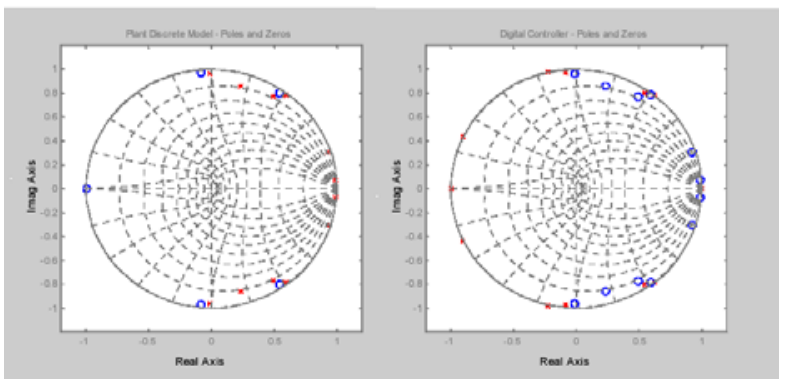

Figura 6. Pólos e Zeros da Planta e Controlador.

O desempenho de controlador foi testado para freqüências de perturbação na gama de 1 a $50 \mathrm{rd} / \mathrm{s}$. Os resultados são apresentados nas Figuras 7 a 10. Pode ser observado que o desempenho do controlador melhora com a diminuição da freqüência da perturbação (como esperado). Note porém que até mesmo para uma freqüência de perturbação de $50 \mathrm{rd} / \mathrm{s}$ (relativamente alta e também uma freqüência ressonante da planta) a atenuação de ruído é maior que $70 \%$ (Figura 7) mostrando um desempenho ainda excelente do controlador. 


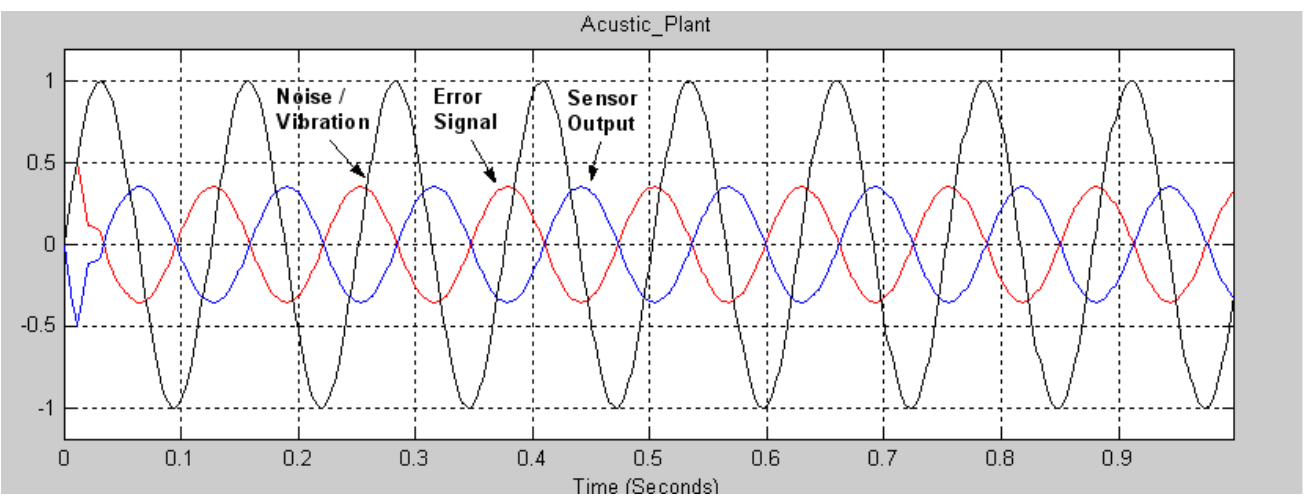

Figura 7. Resposta do Sistema em Malha Fechada para uma Perturbação Senoidal com Wd $=50 \mathrm{rd} / \mathrm{s}$ (pior caso).

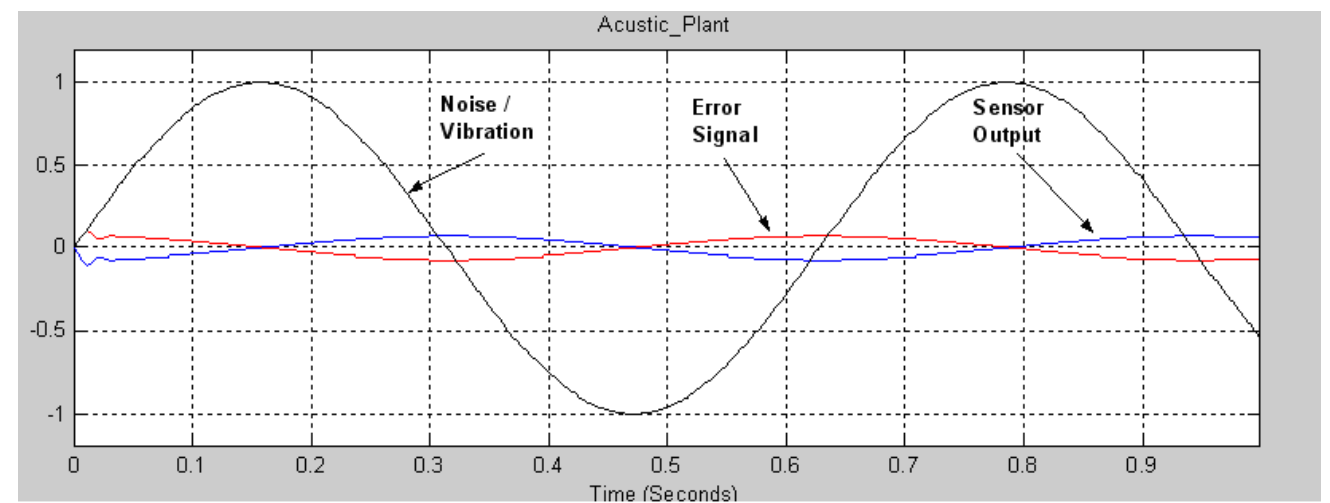

Figura 8. Resposta do Sistema em Malha Fechada para uma Perturbação Senoidal com Wd = $10 \mathrm{rd} / \mathrm{s}$.

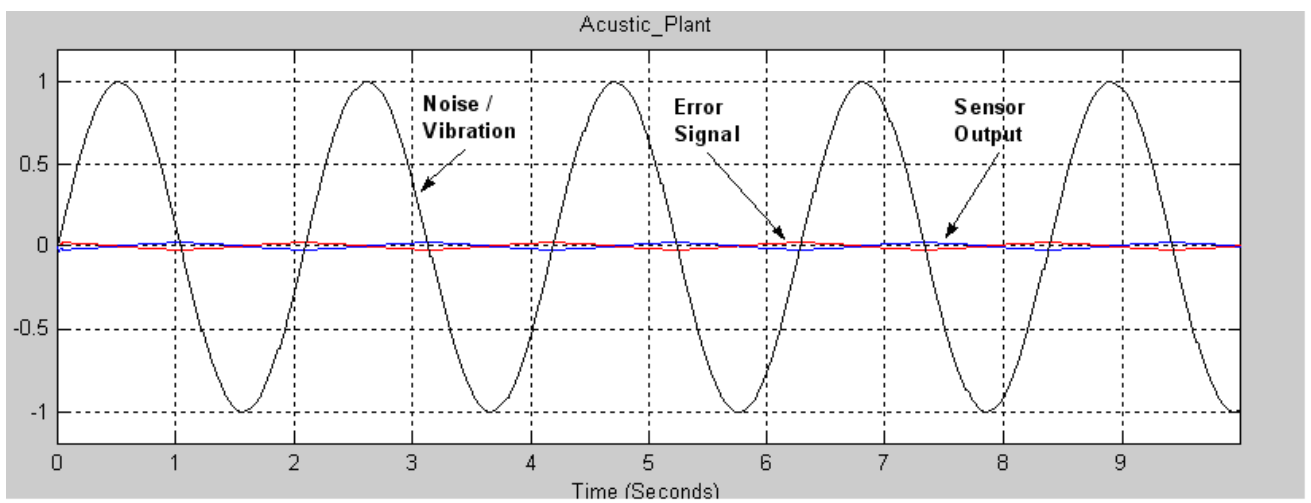

Figura 9. Resposta do Sistema em Malha Fechada para uma Perturbação Senoidal com Wd = 3 rd/s.

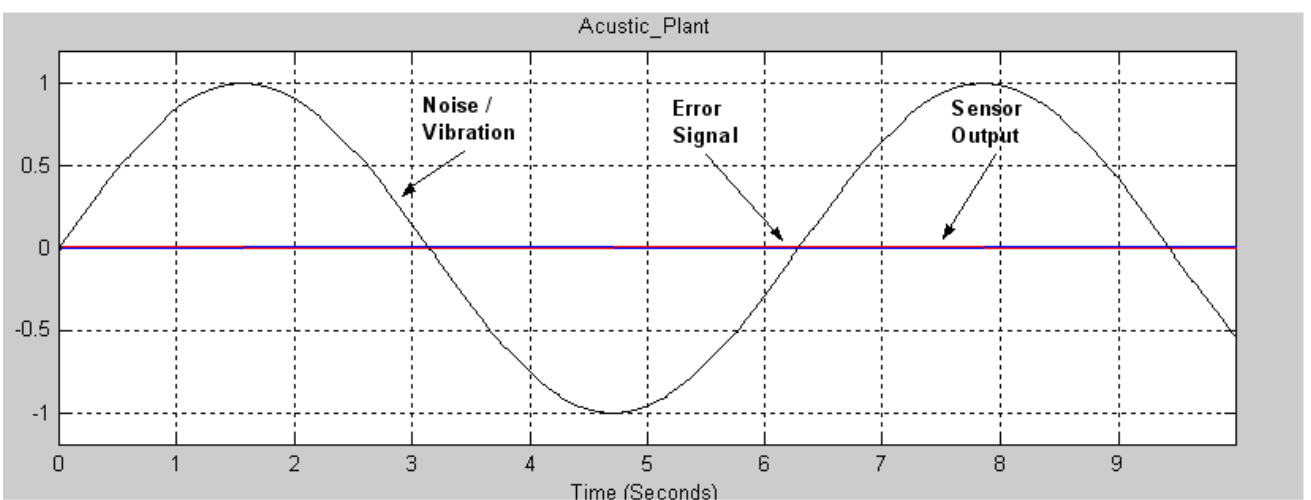

Figura 10. Resposta do Sistema em Malha Fechada para uma Perturbação Senoidal com Wd $=1 \mathrm{rd} / \mathrm{s}$ (melhor caso). 


\section{Comentários Finais e Conclusões}

Um novo procedimento de controle foi apresentado como uma alternativa para o controle ativo de ruído e vibrações. A técnica proposta está baseada no algoritmo de controle deadbeat. $O$ algoritmo foi aplicado a um modelo de $12^{\mathrm{a}}$ ordem de um guia de onda acústico unidimensional com freqüências ressonantes de 50, 212, 617, 669, 872 e $1060 \mathrm{rd} / \mathrm{s}$, a taxa amostragem foi escolhida como sendo $1.5 \mathrm{~ms}$. O algoritmo proposto mostrou excelente desempenho para perturbações de baixa freqüência de até $50 \mathrm{rd} / \mathrm{s}$ produzindo uma redução de ruído de cerca de $70 \%$ nessa freqüência. Finalmente, cabe mencionar que quanto menor a freqüência da perturbação pior seriam os resultados das técnicas passivas e o melhor seria o desempenho de técnica proposta. Este fato mostra uma característica promissora desta técnica em controladores híbridos, Chen et al (2009).

\section{Agradecimentos}

À FAPEMIG pelo apoio financeiro.

\section{Referências Bibliográficas}

Barron, R.F., 2003, "Industrial Noise Control and Acoustics", Marcel Dekker, Inc.

Beranek, L.L., 1996, "Acoustics", Acoustical Society of America - American Institute of Physics, New York.

Beranek, L.L., Vér, I.L., 1992, "Noise and Vibration Control Engineering”, John Wiley \& Sons, INC.

Bistafa, S.R., 2006, "Acústica Aplicada ao Controle de Ruído", Editora Edgard Blücher Ltda, $1^{\text {a }}$ Edição.

Burgess, J.C., 1981, "Active Adaptive Sound Control in a Duct: A Computer Simulation", Journal of the Acoustic Society of America, Vol. 70, No. 3, pp. 715-726. DOI: $10.1121 / 1.386908$

Cabell, R.H., Fuller, C.R., 1999, “A Principal Component Algorithm for Feedforward Active Noise and Vibration Control". Journal of Sound and Vibration, Vol. 227, No. 1, pp. 159-181. DOI: $10.1006 /$ jsvi.1999.2338

Chen, C.T., 1984 "Linear Systems Theory and Design".

Chen, K.T., Chen, Y.N., Chen, W., Liu, Y.H., 1998, "Adaptive Active Control on the Acoustics Transmission of the Acoustic Sources in Aperture at Low Frequencies", Applied Acoustics, Vol. 54, No. 2, pp. 141-164. DOI: $10.1016 / \mathrm{S} 0003-682 \mathrm{X}(97) 00038-8$

Chen, S.R, Too, G.P.J., 2009, "Simulations and Experiments for Hybrid Noise Control Systems",

Applied Acoustics, Vol. 70, No. 1, pp. 247-255. DOI: 10.1016/j.apacoust.2008.04.005

Cremer, L., 1953, "Theory of Attenuation of Airborne Sound in a Rectangular Duct with Sound-absorbing Walls and Maximum Achievable Attenuation", Acustica, Vol. 3, pp. 249-263.

DOI: 10.5540/03.2013.001.01.0198
Crolla, D.A., Abdel Hady, M.B.A., 1991, "Active Suspension Control; Performance Comparisons using Control Laws Applied to a Full Car Model", Vehicle System Dynamics 20, 107-120. DOI: $10.1080 / 00423119108968982$

Cuesta, M., Cobo, P., 2000, "Active Control of the Exhaust Noise Radiated by an Enclosed Generator", Applied Acoustics, Vol. 61, pp. 83-94.

DOI: $10.1016 / \mathrm{S} 0003-682 \mathrm{X}(99) 00062-6$

Donadon, L.V., Arruda, J.R.F., 2005, “Experimental Energetic Analyses of an Actively Controlled OneDimensional Acoustic Waveguide", Journal of Sound and Vibration 280, 159-179. DOI: 10.1016/j.jsv.2003.12.024

Dorf, R.C., Bishop, R.H., 2005, "Modern Control Systems", Pearson Prentice Hall.

Elliott, S.J., Nelson, P. A., 1992, "Active Control of Sound", Academic Press.

Elliott, S.J., 2001, "Signal Processing for Active Noise Control", Academic Press.

Elliott, S.J., Nelson, P.A., 1993, “Active Noise Control”, IEEE Signal Process Magazine, Vol. 10, pp. 12-35. DOI: 10.1109/79.248551

Eriksson, L.J., 1991, "Development of the Filtered-

U Algorithm for Active Noise Control", Journal of the Acoustical Society of American, Vol. 89(1), pp. 857-865. DOI: 10.1121/1.400508

Friot, E., 2006, "Infinite Non-Causality in Active Cancellation of Random Noise", Journal of Sound and Vibration, Vol. 290, pp. 597-618. DOI: 10.1016/j.jsv.2005.04.001

Fujita, T., 1997, "Smart Structure for Active Vbration Control of Building", Proc. of ACTIVE 97, Budapest-Hungary, pp.XIX-XXXVIII.

Fuller, C.R., Von Flotow, A.H., 1995, "Active Control of Sound and Vibration", IEEE Control System, December, pag. 9-19.

Fuller, C.R., Elliott, S.J., Nelson, P.A., 1996, “Active Control of Vibration", Academic Press.

Gerges, S.N.Y., 1992, "Ruido - Fundamentos e Controle", Imprensa Universitária da Universidade Federal de Santa Catarina, Florianópolis, Santa Catarina, Brasil.

Hansen, C, 1996, "Active Control of Noise and Vibration", Cambridge University Press.

Hansen, C.H., Snyder, S.D., 1997, “Active Control of Noise and Vibration”, E\&FN Spon, London.

Kailath, T., 1980, "Linear Systems”, Prentice Hall.

Kuo, S.M., Morgan, D.R., 1996, “Active Noise Control Systems", John Wiley \& Sons, Brisbane, Australia.

Nelson, P.A., Elliott, S.J., 1992, "Active Control of Sound", Academic Press.

Pota, H.R., Kelkar, A.G., 2001, "Modeling and Control of Acoustic Ducts", Transactions of ASME Journal of Vibration and Acoustics, ASME, Vol. 123, pp. 2-10.

Silvester, B.C., 1966, "Vibration Reduction in Motor Cars", Soc. Environmental Engineers 4.

Stein, G.J., Ballo, I., 1991, “Active Vibration Control System for the Driver's Seat for Off-Road Vehicles", Vehicle System Dynamics 20, 57-78. DOI: $10.1080 / 00423119108968980$ 